\title{
Periostin expression induced by oxidative stress contributes to myocardial fibrosis in a rat model of high salt-induced hypertension
}

\author{
HAN WU ${ }^{1 *}$, LIANG CHEN $^{2 *}$, JUN XIE $^{1}$, RAN LI $^{1}$, GUAN-NAN LI ${ }^{1}$, QIN-HUA CHEN ${ }^{1}$, \\ XIN-LIN ZHANG ${ }^{1}$, LI-NA KANG ${ }^{1}$ and BIAO XU ${ }^{1}$ \\ Departments of ${ }^{1}$ Cardiology and ${ }^{2}$ Gynaecology and Obstetrics, Drum Tower Hospital, \\ Nanjing University Medical School, Nanjing, Jiangsu 210008, P.R. China
}

Received June 2, 2015; Accepted May 9, 2016

DOI: $10.3892 / \mathrm{mmr} .2016 .5308$

\begin{abstract}
Periostin is an extracellular matrix protein involved in fibrosis. The present study investigated the importance of periostin in hypertension-induced myocardial fibrosis. Rats were randomly divided into either the normal group $(0.4 \%$ $\mathrm{NaCl}$ diet; $\mathrm{n}=8)$ or hypertension group ( $8 \% \mathrm{NaCl}$ diet; $\mathrm{n}=8)$. For 36 weeks, the blood pressure and heart rate of the rats were monitored. At week 36, the hearts were extracted for further analysis. Masson's staining and western blotting were performed to determine the levels of periostin protein expression, oxidative stress and fibrosis. In addition, fibroblasts were isolated from adult rats and cultured in vitro, and following treatment with angiotensin II (Ang II) and N-acetyl-L-cysteine (NAC), western blotting, immunofluorescence and 2',7' dichlorodihydrofluorescin staining were performed to examine reactive oxygen species production, and periostin and $\alpha$-smooth muscle actin ( $\alpha$-SMA) expression levels. The results demonstrated that periostin expression and oxidative stress were increased in hypertensive hearts compared with normal hearts. The in vitro experiments demonstrated that Ang II upregulated the expression levels of periostin and $\alpha$-SMA compared with the control, whereas, pretreatment with NAC inhibited oxidative stress, periostin and $\alpha$-SMA expression in fibroblasts. In conclusion, the results of the current study suggested that oxidative stress-induced periostin is involved in myocardial fibrosis and hypertension. The present study demonstrated that periostin inhibition may be a promising
\end{abstract}

Correspondence to: Mr. Biao Xu, Department of Cardiology, Drum Tower Hospital, Nanjing University Medical School, 321 Zhongshan Road, Nanjing, Jiangsu 210008, P.R. China

E-mail:xubiao@medmail.com.cn

*Contributed equally

Key words: periostin, hypertension, myocardial fibrosis, oxidative stress approach for the inhibition of hypertension-induced cardiac remodeling.

\section{Introduction}

Numerous large-scale clinical studies have suggested that hypertension is a risk factor for cardiovascular diseases (1-3). High blood pressure promotes the development of cardiovascular diseases via various pathological processes, including endothelial dysfunction (4), inflammation (5) and myocardial fibrosis $(5,6)$. Though the pathogenesis of myocardial fibrosis in hypertension has previously been heavily investigated, the mechanism remains to be fully elucidated.

Periostin is a $90-\mathrm{kDa}$ secretory protein that was previously demonstrated to be secreted from a mouse osteoblastic cell line (7). As an extracellular matrix (ECM) protein, periostin is prominently expressed during tissue fibrosis in the kidney $(8-10)$, lung $(11,12)$ and heart $(13,14)$. The heart ventricles are rich in ECM secreted by fibroblasts, thus, it has been hypothesized that periostin is involved in hypertension-induced myocardial fibrosis.

Previous studies indicate that oxidative stress is involved in the development of hypertension-induced myocardial fibrosis (15-17), however, the exact mechanisms by which oxidative stress induces interstitial fibrosis remain to be elucidated. Multiple lines of evidence have indicated that oxidative stress accelerates fibrosis via regulation of profibrotic factors and ECM, thus, the present study hypothesizes that oxidative stress may contribute to periostin expression. The present study aimed to determine the effect of periostin in myocardial fibrosis in an experimental hypertensive rat model and to investigate the underlying molecular mechanisms.

\section{Materials and methods}

Experimental animals and treatment. The experimental and feeding protocols were approved by the Ethics Committee of Drum Tower Hospital (Nanjing, China). Rats (4 weeks old, male, $\mathrm{n}=16$; weight, 90-115 g) were housed together in an animal room at $22^{\circ} \mathrm{C}$, normal atmosphere and $12 \mathrm{~h}$ light/dark 
cycles, with free access to normal drinking water and fed chow with $0.4 \% \mathrm{NaCl}$ (normal group; $\mathrm{n}=8$ ) or $8 \% \mathrm{NaCl}$ (hypertension group; $n=8$ ) for 36 weeks. At the end of the study, the rats were anesthetized with $3 \%$ isoflurane and 8 hearts from each group were rapidly excised and divided into three parts. Cardiac fibroblasts used in present study were isolated from another 10 normal rats. Physiological parameters, including blood pressure and heart rate, were monitored every 4 weeks (BP-2006A; Softron, Tokyo, Japan) until the animals were sacrificed.

Dihydroethidium (DHE) staining and Masson's staining. At the endpoint of the experiment, the hearts were rapidly excised from the rats and were divided into three parts. One part was treated routinely and the others were flash frozen. The tissue was fixed and embedded with optimal cutting temperature compound (Department of Pathology, Drum Tower Hospital, Nanjing. China), and was subsequently cut into $5 \mu \mathrm{m}$ sections. The frozen heart sections were then incubated with $4 \mu \mathrm{m} / \mathrm{ml}$ DHE dye (Beyotime Institute of Biotechnology, Haimen, China) for $30 \mathrm{~min}$ at $37^{\circ} \mathrm{C}$. Oxidative stress was indicated by red staining and visualized using a fluorescence microscope. Image Pro Plus 6.0 software (Media Cybernetics, Inc., Rockville, MD, USA) was used to quantify the red staining in three randomly selected fields in each section $(n=16)$. The second part of the tissue samples were fixed with $4 \%$ paraformaldehyde, embedded in wax and cut into $5-\mu \mathrm{m}$ sections. Masson's staining was used to assess myocardial fibrosis. Collagen volume fraction was analyzed using Image Pro Plus 6.0 software to quantify the collagen deposition in the heart tissue. The remaining tissue was used for western blot analysis.

Isolation of fibroblasts. Cardiac fibroblasts were isolated from adult rats as previously described (18). Briefly, following anesthesia with $3 \%$ isoflurane of the rats, the hearts were rapidly excised, rinsed and homogenized in cold phosphate-buffered saline (PBS). Then, the tissues were digested in Dulbecco's modified Eagle's medium (DMEM; Hyclone, Logan, TX, USA) containing $0.1 \%$ collagenase type II (Nanjing SunShine Biotechnology Co., Ltd., Nanjing, China) at $37^{\circ} \mathrm{C}$. The resulting supernatant was centrifuged at $36^{\circ} \mathrm{C}$ for $30 \mathrm{~min}$ at $500 \mathrm{x} \mathrm{g}$ and resuspended, prior to seeding in DMEM containing $10 \%$ fetal bovine serum (Hyclone). After $1 \mathrm{~h}$, the supernatant was removed and the adherent cells were maintained as cardiac fibroblasts. Unless otherwise stated, cardiac fibroblasts at passage 1 to 3 , cultured at $37^{\circ} \mathrm{C}$ and $5 \% \mathrm{CO}_{2}$, were used in the present study and were serum-starved overnight prior to treatments with Ang II (1, 2 or $4 \mu \mathrm{M}$; Sigma-Aldrich, St. Louis, MO, USA) or NAC (5 or $10 \mathrm{nM}$; Beyotime Institute of Biotechnology, Haimen, China) for $24 \mathrm{~h}$.

Immunofluorescence for $\alpha$-smooth muscle actin ( $\alpha$-SMA). When adherent cells reached $80-90 \%$ confluency, they were seeded in 24-well plates. Following treatment with Ang II $(4 \mu \mathrm{M})$ or NAC $(10 \mathrm{nM})$ for $24 \mathrm{~h}$, the fibroblasts were washed with PBS 3 times and fixed with $4 \%$ paraformaldehyde for $60 \mathrm{~min}$ at room temperature. After blocking with $2 \%$ BSA at room temperature for $1 \mathrm{~h}$, the cells were incubated with rabbit anti- $\alpha$-SMA primary antibody (cat. no. ab5694; 1:100;
Abcam, Cambridge, UK). Following two washes with PBS, the cells were incubated with Alexa Fluor ${ }^{\circledR}$ 594-conjugated goat anti-rabbit secondary antibody (cat. no. A-11037; 1:400; Invitrogen; Thermo Fisher Scientific, Inc., Waltham, MA, USA) for $1 \mathrm{~h}$ under dark conditions at room temperature. Finally, the cells were counterstained with 4',6-diamidino-2-phenylindole (Sigma-Aldrich, St. Louis, MO, USA) for $10 \mathrm{~min}$, and then visualized using a confocal laser scanning microscope.

Measurement of intracellular reactive oxygen species (ROS) in fibroblasts. Intracellular ROS generation was detected using the fluorescent probe 2',7'-dichlorofluorescin diacetate (DCFH). Briefly, fibroblasts were plated in 24-well plates. Following treatment with Ang II or NAC, the medium was removed and the cells were washed with PBS. A solution of $5 \mu \mathrm{M}$ DCFH probe in serum-free DMEM was then added to the cells for $30 \mathrm{~min}$ at $37^{\circ} \mathrm{C}$. The plates were washed twice and the intracellular ROS levels were detected by microscope.

Western blotting. Tissue or treated cells were homogenized in lysis buffer containing a 1:100 dilution of protease inhibitor (Sigma-Aldrich). The lysates were centrifuged at 13,800 x g for $10 \mathrm{~min}$ and proteins were harvested from the supernatants. Protein concentration was detected using a bicinchoninic acid protein assay kit (Pierce, Rockford, IL, USA). Equal quantities of proteins $(30 \mu \mathrm{g})$ were separated by $10 \%$ SDS-PAGE and electroblotted to polyvinylidene fluoride membranes. Following blocking with $5 \%$ milk, the membranes were incubated with primary antibodies overnight at $4^{\circ} \mathrm{C}$. The membranes were washed with Tris-buffered saline containing $0.1 \%$ Tween 20 then incubated with secondary antibody (cat. no. sc-2004; 1:10,000; Santa Cruz Biotechnology, Inc., Dallas, TX, USA) for $2 \mathrm{~h}$ at room temperature. Proteins were detected using enhanced chemiluminescence reagents (Pierce) and images were obtained by exposure to films. The densities of each band were analyzed using Bio-Rad Quantity One v4.62 imaging software (Bio-Rad Laboratories, Inc., Hercules, CA USA). The results are presented as a percentage change compared with that in controls following normalization to the $\beta$-actin bands for each sample. The following antibodies were used: Anti- $\alpha$-SMA (cat. no. ab5654; 1:1,000; Abcam); anti- $\beta$-actin (cat. no. sc-130657; 1:2,000; Santa Cruz Biotechnology, Inc.), anti-periostin (cat. no. sc-67233; 1:500; Santa Cruz Biotechnology, Inc.), anti-p47phox (cat. no. A1148; 1:1,000; ABclonal Biotech Co., Ltd., Cambridge, MA, USA), anti-gp91phox (cat. no. sc-20782; 1:1,000; Santa Cruz Biotechnology, Inc.), and anti-p66shc (cat. no. sc-1695; 1:1,000; Santa Cruz). All blots were performed on three independent occasions.

Statistical analysis. Analyses were performed using SPSS software (version 21.0; IBM SPSS, Armonk, NY, USA). Differences between 2 groups were analyzed by Student's t-test and one-way analysis of variance was used to test significance for $>2$ groups. Student-Neuman-Keuls or Dunnett's T3 tests were used for post-hoc multiple comparisons. $\mathrm{P}<0.05$ was considered to indicate a statistically significant difference. 
A

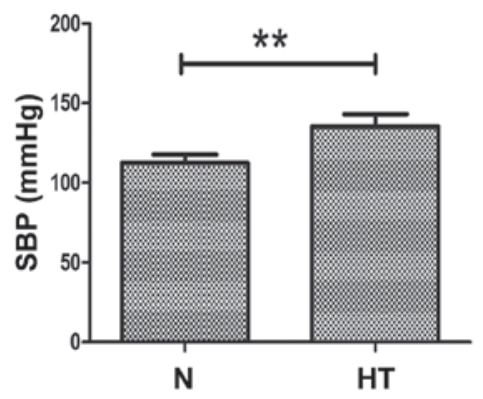

C

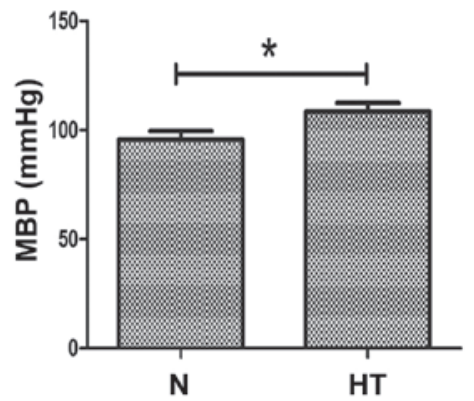

B

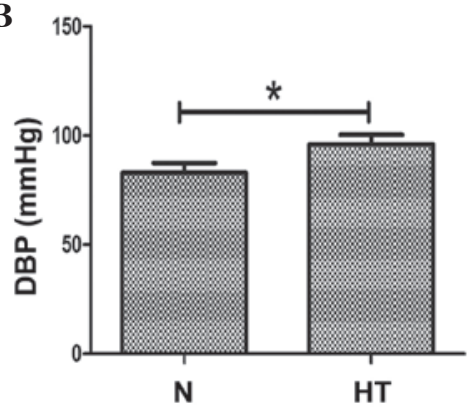

$\mathbf{D}$

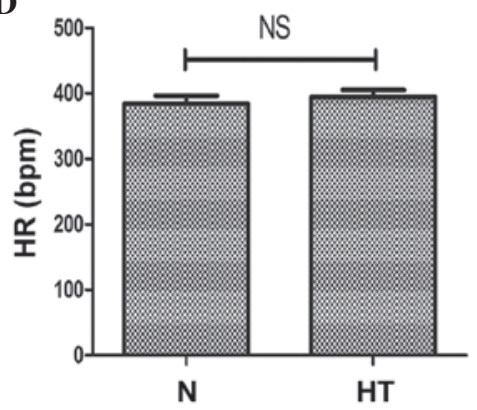

Figure 1. Evaluation of SBP, DBP, MBP and HR in normal and high-salt induced hypertensive rats. (A) SBP, (B) DBP and (C) MBP measurements in high salt-induced hypertensive and normal rats. (D) HR in normal and hypertensive rats. $\mathrm{n}=8$ per group, ${ }^{,} \mathrm{P}<0.05,{ }^{* *} \mathrm{P}<0.01$, comparisons indicated by brackets. $\mathrm{N}$, normal rats group; HT, hypertensive rats group; SBP, systolic blood pressure; DBP, diastolic blood pressure; MBP, mean blood pressure; HR, heart rate; NS, not significant.

A
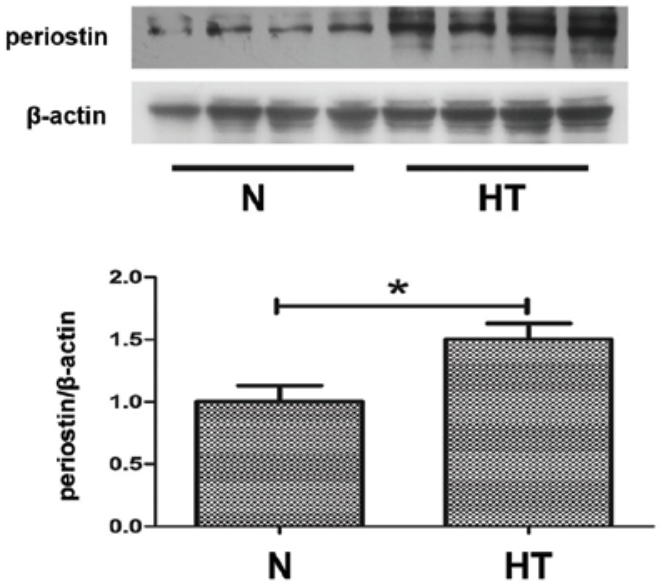

B

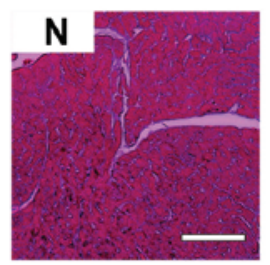

\section{HT}

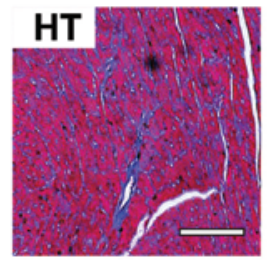

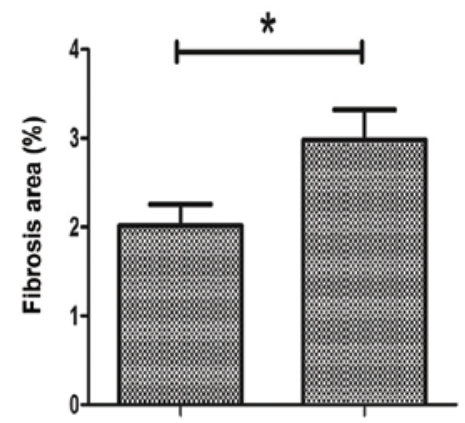

N
HT

Figure 2. Expression of periostin and myocardial fibrosis in hypertensive rats. (A) Representative western blot of periostin in normal and hypertensive hearts. (B) Masson staining in heart sections of the two groups; bar $=100 \mu \mathrm{m}$. "P<0.05, comparisons indicated by brackets. N, normal rats group; HT, hypertensive rats group.

\section{Results}

Blood pressure increased in high-salt induced hypertensive rats. As a high salt diet is an important risk factor in hypertension, an in vivo hypertension model was produced with a sustained high salt diet for 36 weeks. At the end of the experimental period, blood pressure levels and heart rate of the animals were measured. Compared with the normal rats (0.4\% NaCl diet), systolic blood pressure ( $\mathrm{P}<0.01$; Fig. 1A), diastolic blood pressure $(\mathrm{P}<0.05$; Fig. $1 \mathrm{~B})$ and mean blood pressure $(\mathrm{P}<0.05$; Fig. $1 \mathrm{C})$ were significantly increased in the high salt $(8 \% \mathrm{NaCl}$ diet)-induced hypertensive rats. At week 36, no significant differences in heart rate were observed between the 2 groups (Fig. 1D).

Periostin is upregulated and myocardial fibrosis observed in hypertensive rats. Periostin has previously been considered to be an important mediator of fibrosis, thus, western blotting was performed to detect the expression levels of periostin in the hearts of the 2 groups. As demonstrated in Fig. 2A, the protein expression levels of periostin in hypertensive hearts was significantly upregulated compared with normal hearts $(\mathrm{P}<0.05)$. Histological observations following Masson's trichrome staining indicated that myocardial fibrosis was 
A
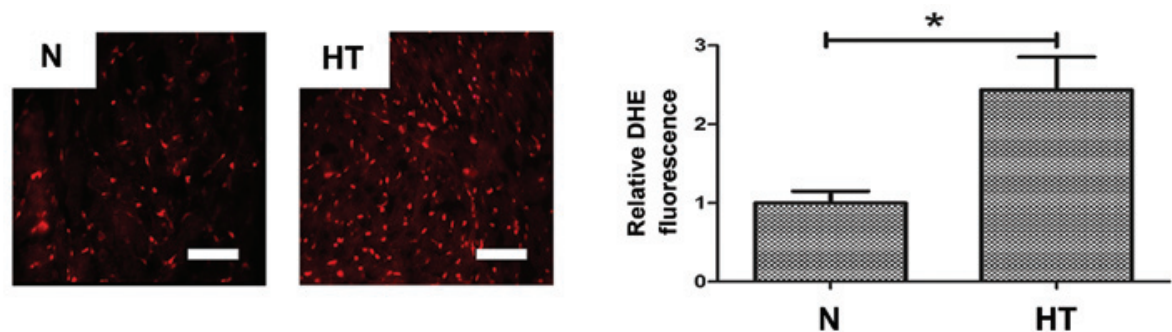

B
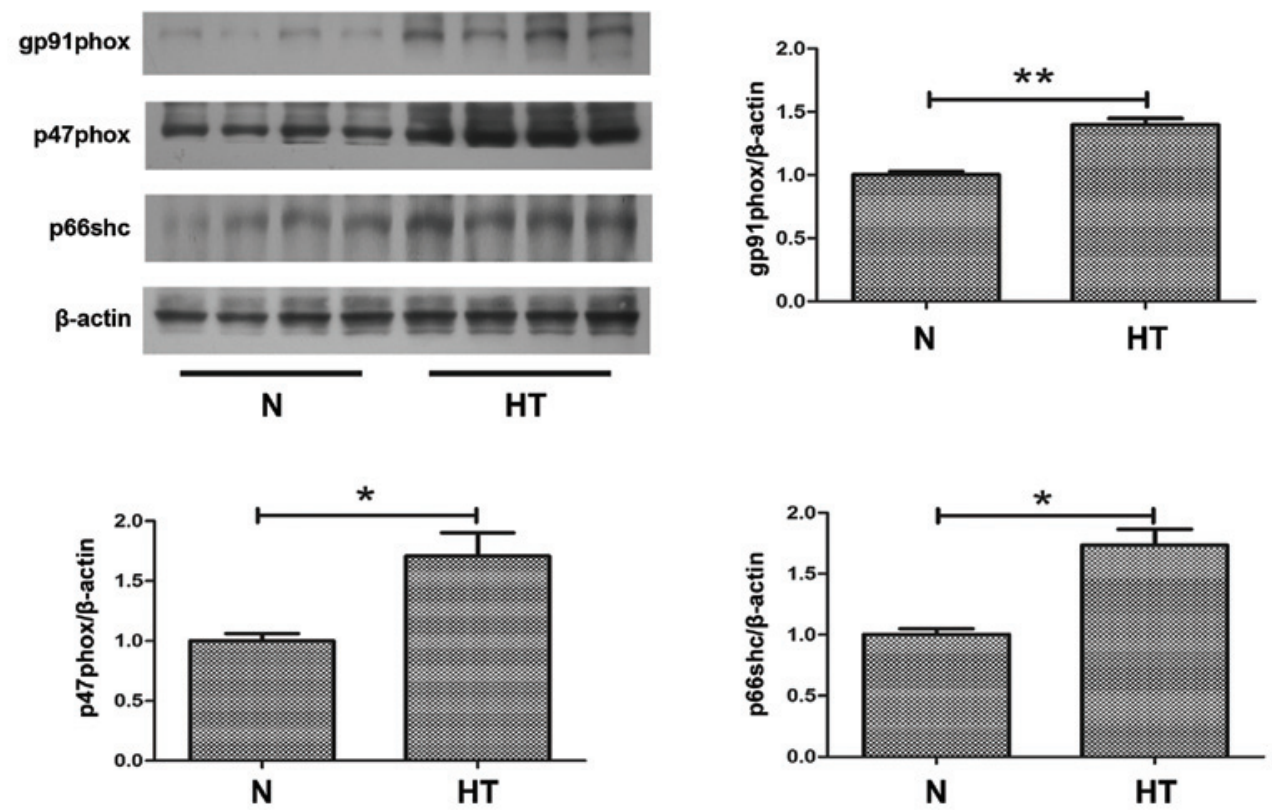

Figure 3. Increased oxidative stress in hypertensive rats. (A) The superoxide level determined by DHE staining in heart sections of the two groups; bar=50 $\mu \mathrm{m}$. (B) Representative western blot of oxidase levels in normal and hypertensive rats. ${ }^{*} \mathrm{P}<0.05,{ }^{* * *} \mathrm{P}<0.01$, comparisons indicated by brackets. $\mathrm{N}$, normal rats group; HT, hypertensive rats group; DHE, dihydroethidium.

induced in hypertensive rats compared with normal rats (P<0.05; Fig. 2B).

Oxidative stress is increased in hypertensive hearts. To investigate the role of oxidative stress in hypertensive fibrosis, ROS production was measured in samples from normal and hypertensive rats. As demonstrated in Fig. 3A, oxidative stress, indicated by DHE staining, was significantly increased in hypertensive hearts compared with normal hearts $(\mathrm{P}<0.05$; Fig. 3A). Furthermore, to evaluate the increased oxidative stress in hypertensive hearts, the expression levels of oxidative stress-associated proteins were determined. Western blotting demonstrated that the protein expression level of gp91phox $(\mathrm{P}<0.01)$, p47phox $(\mathrm{P}<0.05)$ and p66shc $(\mathrm{P}<0.05)$ were significantly increased in the hearts of hypertensive rats compared with normal rats (Fig. 3B). These proteins all contribute to oxidative stress.

Angiotensin II (Ang II) increases expression of periostin and $\alpha$-SMA in cardiac fibroblasts via oxidative stress. Ang II is important for the initiation of myocardial fibrosis, thus, in vitro studies using Ang II to treat cardiac fibroblasts were performed to investigate oxidative stress and periostin expression levels in hypertensive fibrosis. As demonstrated in Fig. 4A, compared with the control group, incubation of fibroblasts with Ang II significantly upregulated the expression of periostin $(\mathrm{P}<0.01)$, whereas the expression was suppressed by pretreating with $\mathrm{N}$-acetyl-L-cysteine (NAC), an inhibitor of $\operatorname{ROS}(\mathrm{P}<0.01$ and $\mathrm{P}<0.05$ for 5 and $10 \mathrm{nM}$ NAC, respectively). The anti-oxidative effect of NAC was demonstrated using a DCFH probe, which indicated that NAC attenuated Ang II-induced ROS production in fibroblasts (Fig. 4B). To investigate the association between periostin and the differentiation of fibroblasts, the expression of $\alpha$-SMA was detected. Western blot analysis (Fig. 4C) and immunofluorescence (Fig. 4D) demonstrated that the increased expression levels of $\alpha$-SMA induced by Ang II compared with control, was abrogated by NAC treatment $(\mathrm{P}<0.01)$.

\section{Discussion}

The current study demonstrated the contribution of oxidative stress-induced periostin to the development of myocardial fibrosis in hypertension.

Hypertensive myocardial fibrosis contributing to myocardial remodeling is the pathophysiological basis of hypertension and eventually results in heart failure. Myocardial fibrosis is considered to be an early event of heart failure by reducing contractile efficiency and demanding greater cardiac contractile force. Fibrosis is a complex condition mediated by profibrotic factors, an imbalance of collagen synthesis and 
A

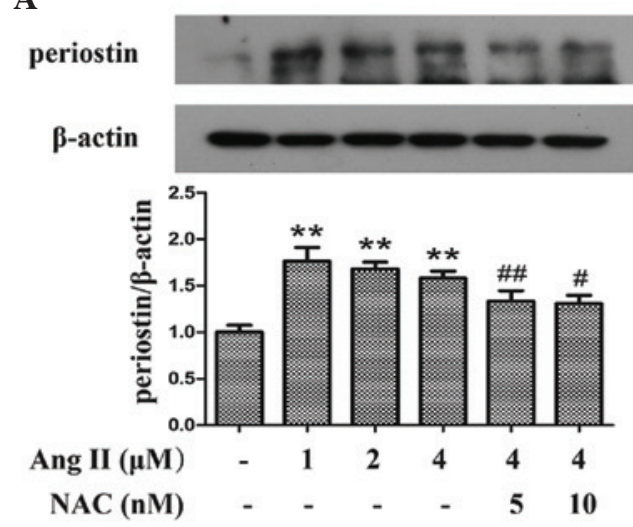

B
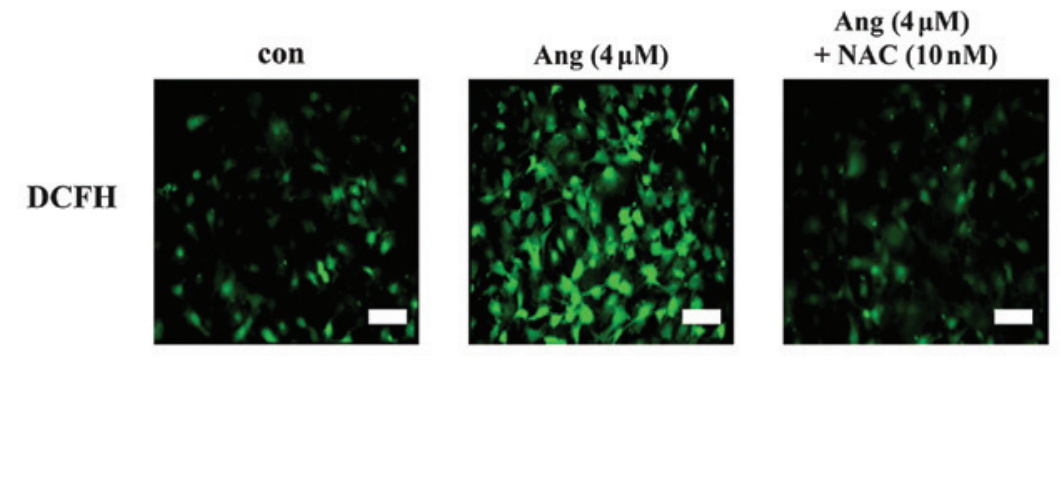

D
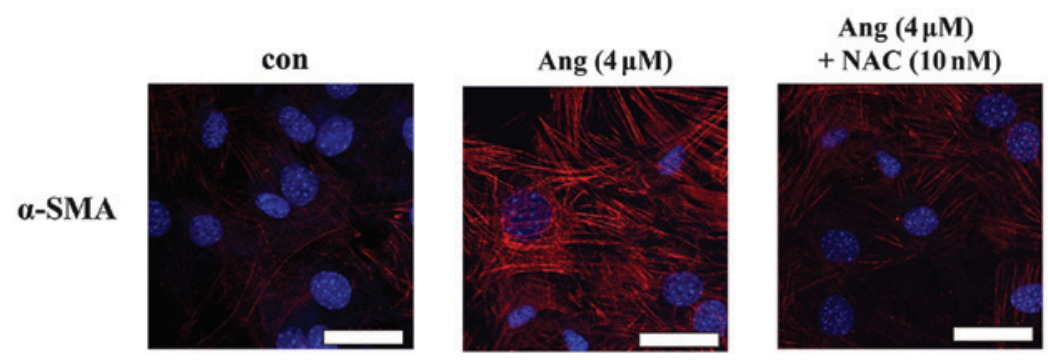

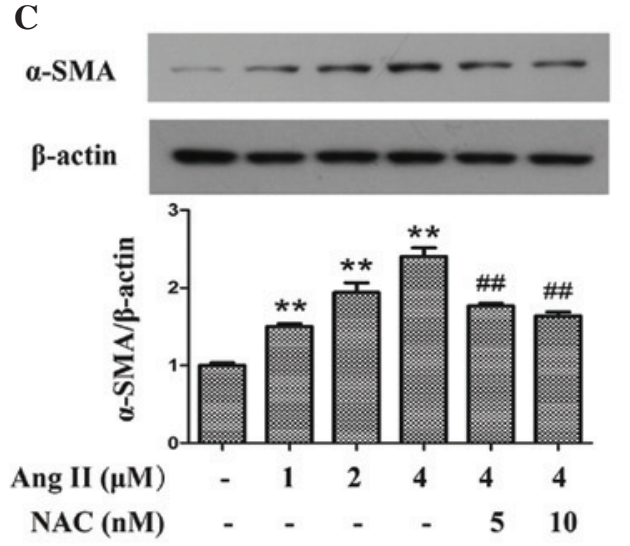

Figure 4. Periostin was induced by oxidative stress in Ang II-induced differentiation of cardiac fibroblasts. (A) In vitro treatment with NAC reduced the expression of perisotin induced by Ang II in cardiac fibroblasts. (B) DCFH staining showed induction of ROS production in Ang II treated fibroblasts, while NAC inhibited ROS genesis. (C) Upregulated expression of $\alpha$-SMA induced by Ang II was decreased by pretreatment of NAC. (D) NAC was observed to attenuate Ang II-induced expression of $\alpha$-SMA using immunofluorescence. ${ }^{* *} \mathrm{P}<0.01$ vs. the control group; ${ }^{\#} \mathrm{P}<0.05$ vs. the Ang II $(4 \mu \mathrm{M})$ group; ${ }^{\# \#} \mathrm{P}<0.01$ vs. the Ang II $(4 \mu \mathrm{M})$ group. Bar=50 $\mu \mathrm{m}$. Ang II, angiotensin II; NAC, N-acetyl-L-cysteine; DCFH, 2',7' dichlorodihydrofluorescin; $\alpha$-SMA, $\alpha$-smooth muscle actin; con, control.

degradation, upregulation of ECM and increased oxidative stress. The present study focused on ECM and oxidative stress in hypertensive hearts.

Increased periostin and oxidative stress in hypertensive rats. Periostin, a member of the fasciclin family, is an ECM protein that promotes progression of fibrosis via regulating ECM homeostasis $(19,20)$. Periostin was previously demonstrated to be upregulated in numerous heart diseases. A previous study by Zhao et al (14) demonstrated that periostin expression was upregulated and associated with myocardial fibrosis in human failing hearts. Another investigation in mice following transverse aortic constriction confirmed the effect of periostin in heart failure (13). Meyer et al (21) demonstrated that periostin expression levels as a biomarker associated with ECM remodeling contributing to heart failure were significantly higher in men compared with women. The results of the present study demonstrated that periostin was also increased in hypertensive hearts. This finding is consistent with another investigation that demonstrated that eplerenone enhanced cardioprotective effects in hypertensive heart failure by regulating ECM proteins, including periostin (22). However, to the best of our knowledge, the mechanisms that regulate periostin expression have not been previously investigated.
Oxidative stress. A major source of ROS in cardiovascular diseases is the nicotinamide adenine dinucleotide phosphate (NADPH) oxidase family (17). NADPH oxidase consists of multiple subunits: Membrane-bound proteins (including gp91phox); cytosolic regulation subunits (such as p47phox); and a small GTP-binding protein Rac 1 (23). The current study demonstrated that $\mathrm{p} 47$ phox and gp91phox were upregulated in hypertensive hearts, which is consistent with the findings of others studies suggesting that NADPH oxidase contributes to kidney and aortic media damage in hypertension $(24,25)$.

In addition, p66shc, an isoform of SRC transforming protein 1, has also been recognized as an important mediator in oxidative stress in the cardiovascular system (26-28). Notably, consistent with previous findings (29), the present study demonstrated that p66shc modulates ROS production in hypertension.

Furthermore, DHE staining was also used to evaluate oxidative stress in hypertensive rats. DHE staining further supported the results of ph47phox, gp91phox and p66shc, suggesting that hypertensive hearts exhibited increased oxidative stress compared with normal hearts. Consistently with the findings of the present study, an accumulating body of evidence suggests that oxidative stress is an essential characteristic of hypertension (30-33). Thus, considering the 
present study and previous findings in hypertension, ROS generation may be important for the development of hypertension.

Ang II increases expression of periostin via oxidative stress. As periostin and oxidative stress were upregulated in hypertension, an in vitro experiment to investigate their association was conducted.

A number of studies have demonstrated that Ang II is the most important hormone that regulates myocardial fibrosis $(18,34)$, which is supported by previous studies that have demonstrated that Ang II antagonists can prevent fibrosis in various diseases $(33,35)$. Ang II promotes systemic arteriole contraction that results in hypertension via binding Ang II type I receptor, and contributes to myofibrosis in hypertension-associated heart diseases. Following stimulation by certain factors, including Ang II, cardiac fibroblasts can differentiate to myofibroblasts, exhibiting increased expression of a number of cytoskeletal protein, including $\alpha$-SMA and collagen. Differentiation of cardiac fibroblasts into myofibroblasts contributes to cardiac fibrosis and eventually affects the dynamic contractile properties of the heart (36). Thus, the present study performed in vitro experiments using cardiac fibroblasts treated with Ang II to investigate the effect of oxidative stress and periostin in hypertensive fibrosis.

The current study demonstrated that Ang II increased the expression of periostin in fibroblasts, which is also supported by the previous finding that periostin was induced by Ang II in cardiac fibroblasts (13). Notably, in the present study, upregulation of periostin was accompanied by an increase in ROS generation, while inhibition of ROS blocked the induction of periostin, suggesting that Ang II upregulates the expression of periostin via an oxidative stress-mediated signaling pathway. To the best of our knowledge, this is the first report demonstrating that oxidative stress also regulates periostin expression induced by Ang II.

Consistent with previous findings, the present study demonstrated that Ang II upregulates the expression of $\alpha$-SMA in fibroblasts by western blot analysis and immunofluorescence (18), suggesting that Ang II induces differentiation of cardiac fibroblasts into myofibroblasts. Notably, the results indicated that ROS-induced periostin expression may be important for Ang II-induced differentiation of cardiac fibroblasts.

In conclusion, the results of the present study demonstrated that oxidative stress-induced upregulation of periostin may be important for in myocardial fibrosis in hypertensive rats. Therefore, periostin may serve as a potential target for the prevention of hypertension-induced myocardial fibrosis.

\section{Acknowledgements}

The current study was supported by grants from the Natural Science Foundation of China (grant nos. 81070195, 81200148 and 81270281), the Jiangsu Key Laboratory for Molecular Medicine of Nanjing University, Jiangsu Provincial Special Program of Medical Science (grant no. BL2012014), the State Key Laboratory of Pharmaceutical Biotechnology (grant no. KF-GN-200901), the Peak of Six Personnel in Jiangsu Province (grant no. 2013-WSN-008), Funds for Distinguished
Young Scientists in Nanjing (grant no. JQX13006), and Natural Science Foundation of Jiangsu Province (grant no. BK2010107).

\section{References}

1. Barasch E, Gottdiener JS, Aurigemma G, Kitzman DW, Han J, Kop WJ and Tracy RP: Association between elevated fibrosis markers and heart failure in the elderly: The cardiovascular health study. Circ Heart Fail 2: 303-310, 2009.

2. Kannan A and Janardhanan R: Hypertension as a risk factor for heart failure. Curr Hypertens Rep 16: 447, 2014.

3. Volpe M, McKelvie R and Drexler H: Hypertension as an underlying factor in heart failure with preserved ejection fraction. J Clin Hypertens (Greenwich) 12: 277-283, 2010.

4. Lapu-Bula R and Ofili E: From hypertension to heart failure: Role of nitric oxide-mediated endothelial dysfunction and emerging insights from myocardial contrast echocardiography. Am J Cardiol 99: 7D-14D, 2007.

5. Palaniyandi SS, Inagaki K and Mochly-Rosen D: Mast cells and epsilonPKC: A role in cardiac remodeling in hypertension-induced heart failure. J Mol Cell Cardiol 45: 779-786, 2008.

6. Mori T, Kai H, Kajimoto H, Koga M, Kudo H, Takayama N, Yasuoka S, Anegawa T, Kai M and Imaizumi T: Enhanced cardiac inflammation and fibrosis in ovariectomized hypertensive rats: A possible mechanism of diastolic dysfunction in postmenopausal women. Hypertens Res 34: 496-502, 2011.

7. Takeshita S, Kikuno R, Tezuka $K$ and Amann E: Osteoblast-specific factor 2: Cloning of a putative bone adhesion protein with homology with the insect protein fasciclin I. Biochem J 294: 271-278, 1993.

8. Wallace DP, White C, Savinkova L, Nivens E, Reif GA, Pinto CS, Raman A, Parnell SC, Conway SJ and Fields TA: Periostin promotes renal cyst growth and interstitial fibrosis in polycystic kidney disease. Kidney Int 85: 845-854, 2014.

9. Bible E: Polycystic kidney disease: Periostin is involved in cell proliferation and interstitial fibrosis in polycystic kidney disease. Nat Rev Nephrol 10: 66, 2014.

10. Mael-Ainin M, Abed A, Conway SJ, Dussaule JC and Chatziantoniou C: Inhibition of periostin expression protects against the development of renal inflammation and fibrosis. J Am Soc Nephrol 25: 1724-1736, 2014.

11. Naik PK, Bozyk PD, Bentley JK, Popova AP, Birch CM, Wilke CA, Fry CD, White ES, Sisson TH, Tayob N, et al: Periostin promotes fibrosis and predicts progression in patients with idiopathic pulmonary fibrosis. Am J Physiol Lung Cell Mol Physiol 303: L1046-L1056, 2012.

12. Okamoto M, Hoshino T, Kitasato Y, Sakazaki Y, Kawayama T, Fujimoto K, Ohshima K, Shiraishi H, Uchida M, Ono J, et al: Periostin, a matrix protein, is a novel biomarker for idiopathic interstitial pneumonias. Eur Respir J 37: 1119-1127, 2011.

13. Liu W, Zi M, Tsui H, Chowdhury SK, Zeef L, Meng QJ, Travis M, Prehar S, Berry A, Hanley NA, et al: A novel immunomodulator, FTY-720 reverses existing cardiac hypertrophy and fibrosis from pressure overload by targeting NFAT (nuclear factor of activated T-cells) signaling and periostin. Circ Heart Fail 6: 833-844, 2013.

14. Zhao S, Wu H, Xia W, Chen X, Zhu S, Zhang S, Shao Y, Ma W, Yang D and Zhang J: Periostin expression is upregulated and associated with myocardial fibrosis in human failing hearts. J Cardiol 63: 373-378, 2014.

15. Redout EM, van der Toorn A, Zuidwijk MJ, van de Kolk CW, van Echteld CJ, Musters RJ, van Hardeveld C, Paulus WJ and Simonides WS: Antioxidant treatment attenuates pulmonary arterial hypertension-induced heart failure. Am J Physiol Heart Circ Physiol 298: H1038-H1047, 2010.

16. Worou ME, Belmokhtar K, Bonnet P, Vourc'h P, Machet MC, Khamis $G$ and Eder V: Hemin decreases cardiac oxidative stress and fibrosis in a rat model of systemic hypertension via PI3K/Akt signalling. Cardiovasc Res 91: 320-329, 2011.

17. Paravicini TM and Touyz RM: NADPH oxidases, reactive oxygen species, and hypertension: Clinical implications and therapeutic possibilities. Diabetes Care 31 (Suppl 2): S170-S180, 2008.

18. Bai J, Zhang N, Hua Y, Wang B, Ling L, Ferro A and Xu B: Metformin inhibits angiotensin II-induced differentiation of cardiac fibroblasts into myofibroblasts. PLoS One 8: e72120, 2013. 
19. Yamaguchi Y: Periostin in skin tissue and skin-related diseases. Allergol Int 63: 161-170, 2014.

20. Liu AY, Zheng H and Ouyang G: Periostin, a multifunctional matricellular protein in inflammatory and tumor microenvironments. Matrix Biol 37: 150-156, 2014.

21. Meyer S, van der Meer P, van Deursen VM, Jaarsma T, van Veldhuisen DJ, van der Wal MH, Hillege HL and Voors AA: Neurohormonal and clinical sex differences in heart failure. Eur Heart J 34: 2538-2547, 2013.

22. Muñoz-Pacheco P, Ortega-Hernández A, Caro-Vadillo A, Casanueva-Eliceiry S, Aragoncillo P, Egido J, Fernández-Cruz A and Gómez-Garre D: Eplerenone enhances cardioprotective effects of standard heart failure therapy through matricellular proteins in hypertensive heart failure. J Hypertens 31: 2309-2318; discussion 2319, 2013

23. Elnakish MT, Hassanain HH, Janssen PM, Angelos MG and Khan M: Emerging role of oxidative stress in metabolic syndrome and cardiovascular diseases: Important role of Rac/NADPH oxidase. J Pathol 231: 290-300, 2013.

24. Tian N, Moore RS, Phillips WE, Lin L, Braddy S, Pryor JS Stockstill RL, Hughson MD and Manning RD Jr: NADPH oxidase contributes to renal damage and dysfunction in Dahl salt-sensitive hypertension. Am J Physiol Regul Integr Comp Physiol 295: R1858-R1865, 2008.

25. Akasaki T, Ohya Y, Kuroda J, Eto K, Abe I, Sumimoto H and Iida M: Increased expression of gp91phox homologues of NAD (P)H oxidase in the aortic media during chronic hypertension: Involvement of the renin-angiotensin system. Hypertens Res 29: 813-820, 2006

26. Lebiedzińska M, Suski J, Duszyński J and Wieckowski MR: Role of the p66Shc protein in physiological state and in pathologies. Postepy Biochem 56: 165-173, 2010 (In Polish).

27. Lee SK, Chung JI, Park MS, Joo HK, Lee EJ, Cho EJ, Park JB, Ryoo S, Irani K and Jeon BH: Apurinic/apyrimidinic endonuclease 1 inhibits protein kinase C-mediated p66shc phosphorylation and vasoconstriction. Cardiovasc Res 91: 502-509, 2011 .
28. Di Stefano V, Cencioni C, Zaccagnini G, Magenta A, Capogrossi MC and Martelli F: P66ShcA modulates oxidative stress and survival of endothelial progenitor cells in response to high glucose. Cardiovasc Res 82: 421-429, 2009.

29. Camici GG, Sudano I, Noll G, Tanner FC and Lüscher TF Molecular pathways of aging and hypertension. Curr Opin Nephrol Hypertens 18: 134-137, 2009.

30. Lin CX, Rhaleb NE, Yang XP, Liao TD, D'Ambrosio MA and Carretero OA: Prevention of aortic fibrosis by $\mathrm{N}$-acetyl-seryl-asp artyl-lysyl-proline in angiotensin II-induced hypertension. Am J Physiol Heart Circ Physiol 295: H1253-H1261, 2008.

31. Rizzi E, Guimaraes DA, Ceron CS, Prado CM, Pinheiro LC, Martins-Oliveira A, Gerlach RF and Tanus-Santos JE: $\beta 1$-Adrenergic blockers exert antioxidant effects, reduce matrix metalloproteinase activity, and improve renovascular hypertension-induced cardiac hypertrophy. Free Radic Biol Med 73: 308-317, 2014.

32. Chen X, Mori T, Guo Q, Hu C, Ohsaki Y, Yoneki Y, Zhu W, Jiang Y, Endo S, Nakayama K, et al: Carbonyl stress induces hypertension and cardio-renal vascular injury in Dahl salt-sensitive rats. Hypertens Res 36: 361-367, 2013.

33. Chaykovska L, Alter ML, von Websky K,Hohmann M, Tsuprykov O, Reichetzeder C, Kutil B, Kraft R, Klein T and Hocher B: Effects of telmisartan and linagliptin when used in combination on blood pressure and oxidative stress in rats with 2-kidney-1-clip hypertension. J Hypertens 31: 2290-2298; discussion 2299, 2013.

34. Xu J, Carretero OA, Liao TD, Peng H, Shesely EG, Xu J, Liu TS, Yang JJ, Reudelhuber TL and Yang XP: Local angiotensin II aggravates cardiac remodeling in hypertension. Am J Physiol Heart Circ Physiol 299: H1328-H1338, 2010.

35. Yamada Y, Tsuboi K, Hattori T, Murase T, Ohtake M,Furukawa M, Ueyama J, Nishiyama A, Murohara T and Nagata K: Mechanism underlying the efficacy of combination therapy with losartan and hydrochlorothiazide in rats with salt-sensitive hypertension. Hypertens Res 34: 809-816, 2011.

36. Leask A: Potential therapeutic targets for cardiac fibrosis: TGFbeta, angiotensin, endothelin, CCN2 and PDGF, partners in fibroblast activation. Circ Res 106: 1675-1680, 2010. 